\title{
Dynamics of crystal formation in the Greenland NorthGRIP ice core
}

\author{
Joaghim MATHIESEN, ${ }^{1}$ Jesper FERKINGHOFF-BORG, ${ }^{1}$ Mogens H. JENSEN, ${ }^{1}$ \\ Mogens LeVInsen, ${ }^{1}$ Poul OLESEN, ${ }^{1}$ Dorthe DAHL-JEnSEN, ${ }^{2}$ Anders SVEnSSON ${ }^{2}$ \\ ${ }^{1}$ The Niels Bohr Institute, Blegdamsvej 17, DK-2100 Copenhagen, Denmark \\ ${ }^{2}$ Department of Geophysics, Niels Bohr Institute for Astronomy, Geophysics, and Physics, Juliane Maries Vej 30, \\ DK-2100 Copenhagen, Denmark \\ E-mail:as@gfy.ku.dk
}

\begin{abstract}
The North Greenland Icecore Project (NorthGRIP) ice core provides palaeoclimatic information back to about $120 \mathrm{kyr}$ BP. The size distributions of ice crystals in the upper $880 \mathrm{~m}$ of the NorthGRIP ice core, which cover a time-span of approximately 5300 years, have been obtained previously. The distributions evolve towards a universal curve, indicating a common underlying physical process in the formation of crystals. We identify this process as an interplay between fragmentation of the crystals and diffusion of their grain boundaries. The process is described by a two-parameter differential equation to which we obtain the exact solution. The solution is in excellent agreement with the measured distributions.
\end{abstract}

\section{INTRODUCTION}

The North Greenland Icecore Project (NorthGRIP) drilling location $\left(75.10^{\circ} \mathrm{N}, 42.32^{\circ} \mathrm{W}\right)$ is situated on an ice ridge where the ice thickness is $3085 \mathrm{~m}$ (Dahl-Jensen and others, 2002). The mean annual temperature is $-32^{\circ} \mathrm{C}$, and the present annual accumulation in the area averages $19.5 \mathrm{~cm}$ ice equivalent. In the upper $80 \mathrm{~m}$ of the ice sheet, the firn, the snow gradually compacts to a close packing of ice crystals of typical sizes $1-5 \mathrm{~mm}$. We study crystal-size distributions obtained from 15 vertical thin sections of ice evenly distributed in the depth interval 115-880 $\mathrm{m}$ (Svensson and others, 2003a). The thin sections have dimensions of $20 \mathrm{~cm} \times 10 \mathrm{~cm}$ (height $\times$ width) and a thickness of $0.4 \pm 0.1 \mathrm{~mm}$. Digital images of ice thin sections placed between crossed linear polarizers are used to map the dimensions of individual ice crystals in the sample (Fig. 1). The ages of the considered samples are all $<5300$ years (Johnsen and others, 2001), and the temperature of the ice in this period can be assumed constant (Dahl-Jensen and others, 1998).

Figure 2 shows size distributions of ice crystals at selected depths down to $880 \mathrm{~m}$. The crystal size is defined as the vertical extent of a crystal, which is estimated as the height of the minimal vertically aligned rectangle that encloses the individual crystal (see Fig. 1 inset). Using the horizontally measured sizes of the ice crystals from the thin sections, similar distributions are obtained. The thin sections used in determining the ice size distributions have typical thicknesses of $0.4 \pm 0.1 \mathrm{~mm}$, and ice crystals with length scales smaller than this thickness will consequently be either smoothed away when cutting the ice or be invisible in the experimental set-up. These small crystals will be seen as part of the larger crystals and not as individual crystals. This corresponds to an average increase in the sizes of the large crystals, i.e. the lengths we measure $x$ are equal to the real length $\hat{x}$ plus some noise $\epsilon$ of positive mean, $x=\hat{x}+\epsilon$.
We assume that this noise is sufficiently peaked around half the thickness of the thin section, such that we simply put $\epsilon=0.2 \mathrm{~mm}$. This value of $\epsilon$ can be observed directly as a cut-off in the measured distributions and has been used whenever we compare to theoretical estimates. In addition, we note that $\epsilon$ might have a weak $x$ dependence, but we expect it to be negligible compared to the experimental uncertainties.

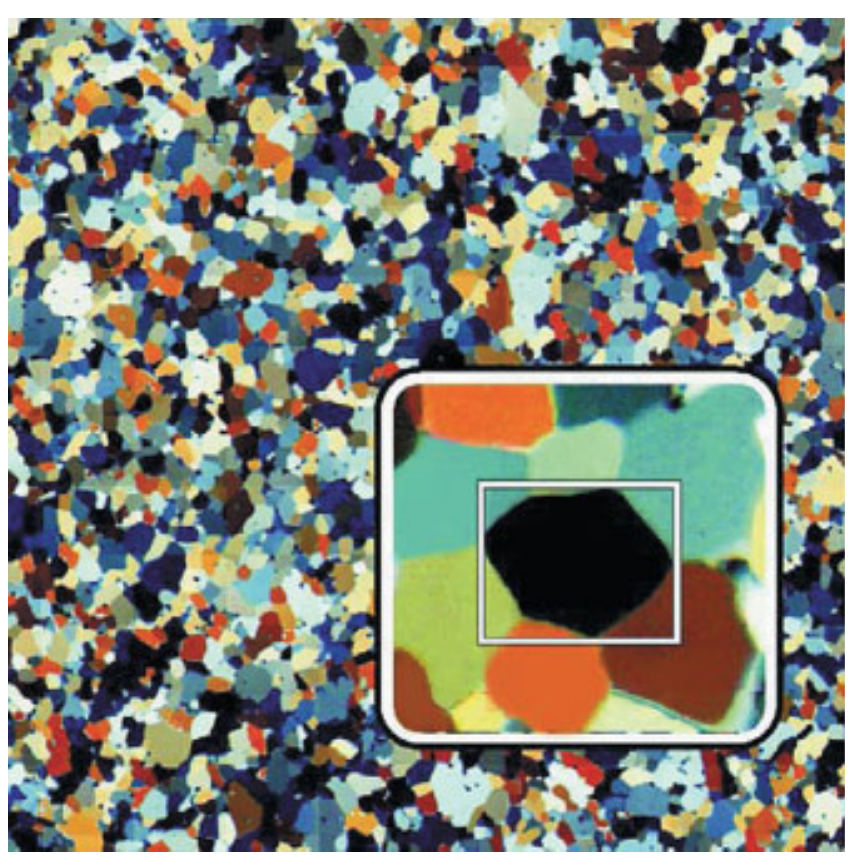

Fig. 1. An image of a $10 \times 10 \mathrm{~cm}^{2}$ vertical thin section of ice from $115 \mathrm{~m}$ depth. The section is viewed between two crossed linear polarizers, and the different colours represent almost 3000 individual crystals with various orientations of the crystal optical c axes. The inset shows an example of the bounding box used when estimating the crystal size. 


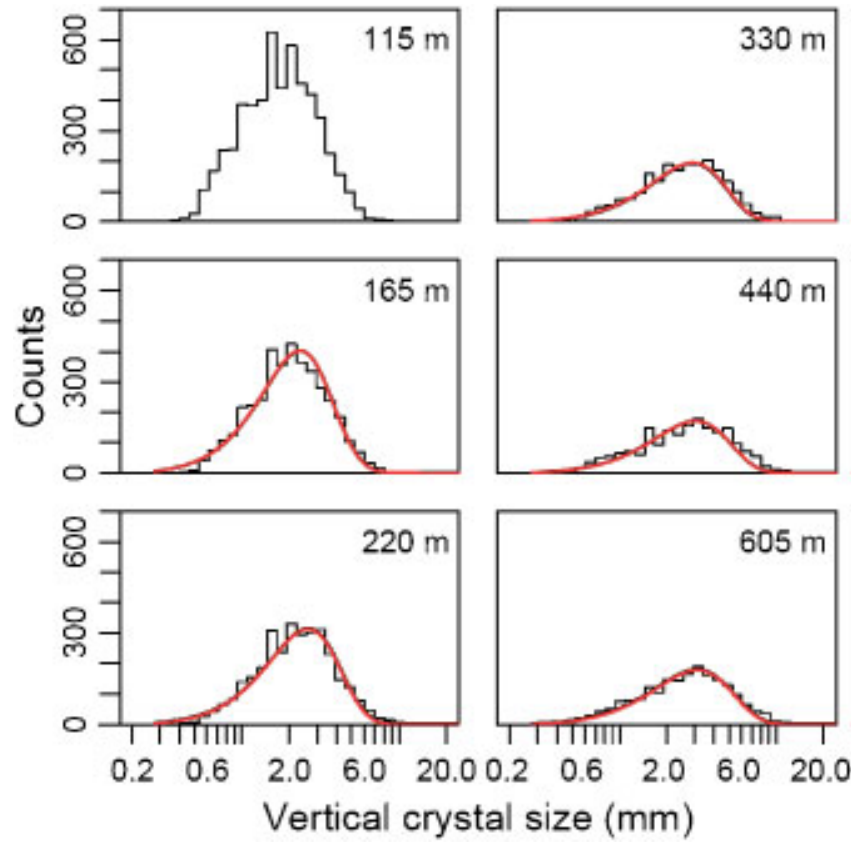

Fig. 2. Distributions of ice crystal sizes at depths 115, 165, 220, 330, 440 and $605 \mathrm{~m}$. The crystal size is defined as the maximum vertical extent of the individual crystals. The black lines are the measured histograms, and the smooth grey lines are the temporal evolution predicted by Equation (2) starting directly from the distribution observed at $115 \mathrm{~m}$. Note that already at $165 \mathrm{~m}$ depth, only a few of the terms in Equation (6) remain, leading to a smooth shape of the predicted distribution. The total number of ice crystals decreases with depth (due to the overall increase in sizes) until the steady state is reached.

Each distribution exhibits a pronounced peak, indicating a typical crystal size at each depth, followed by an exponentially decaying tail of relatively large crystals. The mean size increases with depth and thus time until it reaches a limiting crystal size, which will vary depending on conditions (Thorsteinsson and others, 1997; Li and others, 1998). The process behind the grain growth can effectively be described in terms of boundary diffusion between the ice crystals (Louat, 1974). In this respect, it is the grain boundaries rather than the grains themselves that have physical significance. The grains, characterized by small radii of curvature, tend to be eliminated by motions toward their centres of curvature as time passes. This causes smaller crystals to be incorporated into the larger crystals, leading to an overall increase in mean crystal size (Alley and others, 1986; Paterson, 1994). This approximative description leads to the socalled normal grain-growth law that predicts the following temporal dependence of the mean crystal size $x$ :

$$
\left\langle x^{2}\right\rangle(t)=\left\langle x_{0}^{2}\right\rangle+k t,
$$

where $x_{0}$ is the initial crystal size and $k$ is the crystal growth rate. This approach was successfully applied for the Greenland Icecore Project (GRIP) ice core by Thorsteinsson and others (1997), who fitted their measurements well back to 2500 BP. By definition, however, diffusion does not cause the crystal size to reach a limiting size, whereas the observations clearly indicate a saturation in $\left\langle x^{2}\right\rangle$ after $2500 \mathrm{BP}$ (Alley and Woods, 1996; Gow and others, 1997; Thorsteinsson and others, 1997).

The important missing part of the physical mechanism in this model is the fact that all crystals, and in particular the large ones, are subjected to fragmentation processes, or what is often referred to in ice physics as polygonization or rotation recrystallization (Alley and others, 1995; Thorsteinsson and others, 1997). We include this process in the description here, which then leads to a physical model with a balance between the diffusion of grain boundaries and the fragmentation of crystals, causing the mean crystal size to reach a steady state after $2500 \mathrm{BP}$.

Another process that has been proposed as important for the production of small crystals is nucleation, or the formation of new grains, which may have $c$-axis orientations that differ from the dominating vertical orientation of the surrounding crystals. Measurements of the NorthGRIP fabric ( $c$-axis orientations) suggest, however, that nucleation does not play an important role in the Holocene (Wang and others, 2002; Svensson and others, 2003a), and therefore nucleation is not included in the model. Models of fabric evolution have also often discussed the process of polygonization and dynamic recrystallization leading to changes in crystal size (see, e.g., Thorsteinsson, 2002 and references therein).

Recently, we have proposed a simple model for diffusion and fragmentation processes in one dimension (FerkinghoffBorg and others, 2003). Here, we elaborate on the physical basis for applying the model to crystal grain growth and further demonstrate the effect of crystal-size saturation and the one-parameter nature of the stationary distribution at large times.

\section{THE MODEL}

Our proposed model is formulated as a rate equation in the quantity $N(x, t)$, which is the density of ice crystals of length $x$ at time $t$ measured BP. In this study, $x$ is chosen as the vertical extent of the crystals. At a given time, $N(x, t)$ can be increased or decreased by diffusion with a diffusion constant $D$. It can receive fragments of size $x$ from fragmentations of larger crystals and it can decrease by its own fragmentation. The fragmentation is defined as a rate $f$ in length and time, i.e. for a given time-step $\mathrm{d} t$ the average number of fragmentation events over a length $L$ is $f L \mathrm{~d} t$.

The fragmentation rate $f$, and the diffusion constant $D$, will depend on temperature, non-hydrostatic stresses in the ice and moreover be sensitive to the impurity content of the ice (Alley and others, 1986).

These diffusion and fragmentation processes lead to an integral-differential equation of the form

$$
\begin{aligned}
\frac{\partial N(x, t)}{\partial t}= & D \frac{\partial^{2} N(x, t)}{\partial x^{2}}-f x N(x, t) \\
& +2 f \int_{x}^{\infty} N\left(x^{\prime}, t\right) \mathrm{d} x^{\prime} .
\end{aligned}
$$

Here, the first term on the righthand side is a diffusion term that corresponds to the grain boundary diffusion of the normal grain-growth law, i.e. if we only include this term in the model we reproduce for large times the parabolic behavior in Equation (1). Note that the equation describes the dynamics of the full assembly of crystals and that the mean square of the crystal sizes therefore follows from

$$
\left\langle x^{2}\right\rangle(t)=\frac{\int_{0}^{\infty} x^{2} N(x, t) \mathrm{d} x}{\int_{0}^{\infty} N(x, t) \mathrm{d} x} .
$$

If we only include the diffusion term in Equation (2), we find that for large times $\left\langle x^{2}\right\rangle(t) \sim 4 D t$. The behaviour at large 


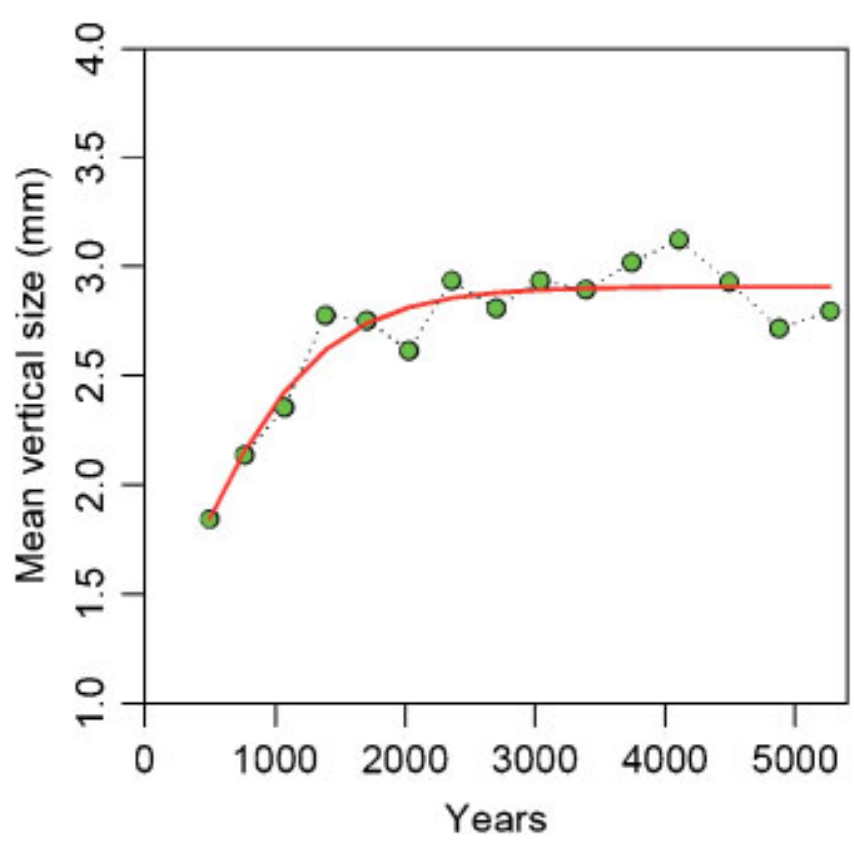

Fig. 3. The mean vertical size of the ice crystals shown vs their age in years BP. The smooth line showes the best fit predicted from our dynamical description of ice crystal growth. From the fit we read off the diffusion constant, $D \approx 1.4 \times 10^{-3} \mathrm{~mm}^{2} \mathrm{a}^{-1}$, and fragmentation rate, $f \approx 5.2 \times 10^{-4} a^{-1} \mathrm{~mm}^{-1}$.

times differs from normal diffusion $\left\langle x^{2}\right\rangle(t) \sim 2 D t$ by a factor of two because we use the absorbing boundary condition that for all times $N(0, t)=0$ (see Louat, 1974, for an explicit solution). This condition expresses the fact that small grains are eliminated and no new crystals will be nucleated.

The second term in Equation (2) is the rate at which crystals of size $x$ fragment, and the last term is the contribution from fragmentation of larger crystals into crystals of size $x$. Combining the two ways a crystal of size $x^{\prime}>x$ can produce a fragment of size $x$ with the assumption that there is a uniform probability, $1 / x^{\prime}$, for where the crystal breaks, we obtain

$$
f x^{\prime} N\left(x^{\prime}\right) \frac{2}{x^{\prime}},
$$

where $f x^{\prime} N\left(x^{\prime}\right)$ is the number of crystals of size $x^{\prime}$ that fragment per time and $2 / x^{\prime}$ is the probability for generating a fragment of size $x$. Note that a uniform probability was already assumed in the definition of the rate $f$. If we integrate Equation (4) over all crystals larger than $x$ we achieve the last term in Equation (2).

Dividing through by $f$ in Equation (2), we obtain an expression on the righthand side which depends on one parameter $a=D / f$ only. The integral-differential equation has analytic solutions, $B\left[(x+\lambda) / a^{1 / 3}\right]$, which are explicitly given in terms of the Bessel function $K_{1 / 3}$ and eigenvalues $\lambda$,

$$
B\left(\frac{x+\lambda}{a^{1 / 3}}\right)=\frac{x^{3 / 2}}{\sqrt{3}} K_{1 / 3}\left[\frac{2}{3}\left(\frac{x+\lambda}{a^{1 / 3}}\right)^{3 / 2}\right] .
$$

The function $K_{1 / 3}(x)$ can be written as a sum of the more frequently used Bessel functions $I(x), K_{1 / 3}(x)=$ $(\pi / \sqrt{3})\left(I_{-1 / 3}(x)-I_{1 / 3}(x)\right)$. The boundary condition, $N(0, t)=0$ for all times $t$, implies that only a discrete set of non-positive values for $\lambda$ is allowed. They are found by solving the equation $B\left(\lambda / a^{1 / 3}\right)=0$. The largest eigen- values are $\lambda_{0} / a^{1 / 3}=0, \lambda_{1} / a^{1 / 3}=-2.338 \ldots, \lambda_{2} / a^{1 / 3}=$ $-4.088 \ldots, \ldots$ The general solution can be written as a linear combination of the eigenfunctions,

$$
N(x, t)=\sum_{n=0}^{\infty} c_{n} B\left(\frac{x+\lambda_{n}}{a^{1 / 3}}\right) \mathrm{e}^{\lambda_{n} f\left(t-t_{0}\right)},
$$

where the $c_{n}$ 's can be computed from the distribution at time $t=t_{0}$ (for further details see Ferkinghoff-Borg and others, 2003). The fact that we have no positive eigenvalues guarantees the existence of a steady-state solution, which is defined by the only surviving term (corresponding to $\lambda_{0}=0$ ) for high ages,

$$
N(x, t) \sim B\left(x / a^{1 / 3}\right) \quad \text { for } \quad t \rightarrow \infty .
$$

The characteristic time, $\tau$, of the exponential growth towards steady state is given by the second largest eigenvalue, $\lambda_{1}$, i.e.

$$
\tau=-\frac{1}{\lambda_{1} f} \approx\left[\left(2.338 f\left(a^{1 / 3}\right)\right]^{-1} .\right.
$$

When the dynamics has reached steady state, and the mean crystal size has saturated, the distribution is described by the single parameter, $a^{1 / 3}$, which defines the characteristic length scale of the system. In particular, the mean vertical size in the steady state is found to be

$$
\langle x\rangle_{\infty}=3^{2 / 3} \frac{\Gamma(4 / 3)}{\Gamma(3 / 2)} a^{1 / 3} .
$$

In Figure 3 we show the mean vertical size of the ice crystals, $\langle x\rangle(t)$ as a function of time. The dots are the measured values, and the solid line is an exponential fit corresponding to the two leading terms in the solution,

$$
\frac{\langle x\rangle_{\infty}}{1+\left(\frac{\langle x\rangle_{\infty}}{\langle x\rangle_{0}}-1\right) \mathrm{e}^{-\left(t-t_{0}\right) / \tau}},
$$

where $\langle x\rangle_{0}$ is the observed average length at time $t_{0}=500$ years. From the figure, we estimate the characteristic time, $\tau=600 \pm 70$ years and the average length in the steady state $\langle x\rangle_{\infty}=2.9 \pm 0.1 \mathrm{~mm}$. The two parameters correspond to an effective fragmentation rate and a diffusion constant of respectively $f=(5.2 \pm 0.6) \times 10^{-4} \mathrm{~mm}^{-1} \mathrm{a}^{-1}$ and $D=(1.4 \pm 0.2) \times 10^{-3} \mathrm{~mm}^{2} \mathrm{a}^{-1}$. Following Equation (3) we obtain the normal grain-growth crystal growth rate $k=4 D=(5.5 \pm 0.8) \times 10^{-3} \mathrm{~mm}^{2} \mathrm{a}^{-1}$, which corresponds very well to the known values for GRIP, $k=$ $5.6 \times 10^{-3} \mathrm{~mm}^{2} \mathrm{a}^{-1}$ (Thorsteinsson and others, 1997), and for NorthGRIP, $k=5.8 \times 10^{-3} \mathrm{~mm}^{2} \mathrm{a}^{-1}$ (Svensson and others, 2003a). We note, however, that the quoted values were obtained from crystal areas and corrected for the sectioning effect with a correction factor of 1.5 , whereas the reported value is obtained from the directly measured maximum vertical extent of the crystals within the thin section.

Using these estimates, we can predict the time evolution of crystal sizes from any initial distribution. We assume that possible variations in the initial distributions for each of the samples can be neglected. We therefore directly use the observed distribution at time $t_{0}=500$ years ( $115 \mathrm{~m}$ depth) as a common initial distribution. The solid lines in Figure 2 show the time evolution of this distribution, in good agreement with the measurements.

When comparing the model to the vertical crystal sizes, we should in principle take into account the vertical compression of crystals due to the ice flow. Had we chosen the horizontal crystal sizes for comparison, we should have 


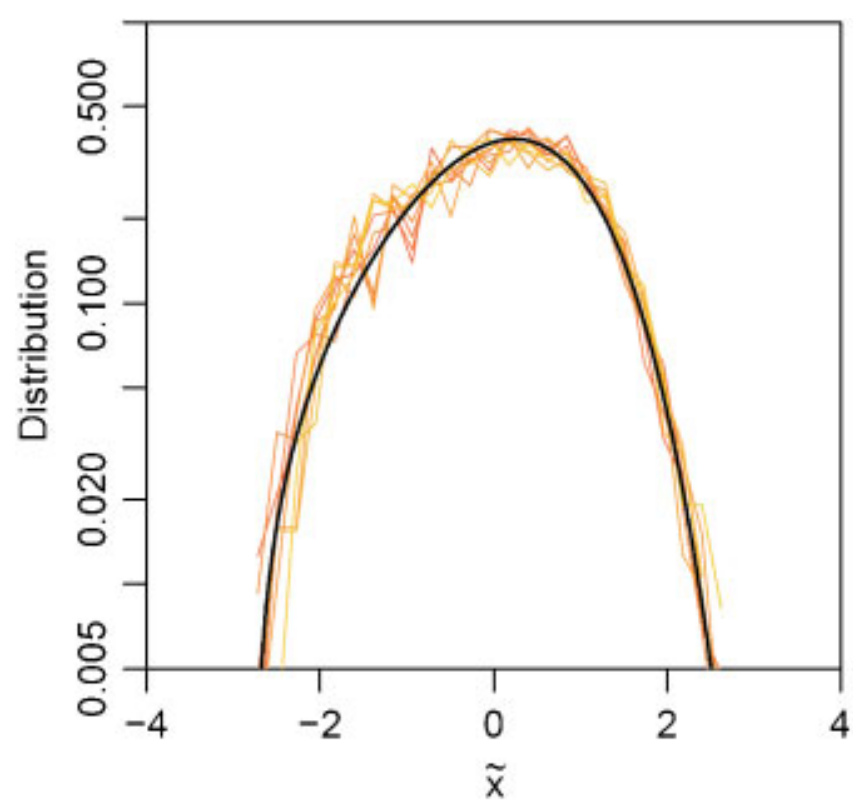

Fig. 4. The figure shows a "data collapse" of the size distributions as a consequence of a rescaling, $\tilde{x}=$ $(\log x-\langle\log x\rangle) / \sigma(\log (x))$, i.e. the distributions shown have zero mean and unit standard deviation. The lines correspond to the distribution used in the eight data points in Figure 3 of the oldest samples ( $t>2500$ years). The black line on top is the steady-state solution of Equation (2). We use the rescaling $\tilde{x}$ in order to improve the resolution around the smallest crystal sizes and note that the steady-state solution is transformed accordingly.

taken into account the corresponding elongation. However, observations show that the crystal flattening, which is defined as crystal width divided by crystal height, is $<15 \%$ for the applied samples (Svensson and others, 2003b), so in order to keep things reasonably simple, we ignore this effect.

\section{DISGUSSION AND GONGLUSIONS}

During an annual cycle, the impurity content and the average crystal size show important variations with the highest dust load and the smallest crystals appearing during spring (Svensson and others, 2003b). Because the size of the applied samples $(20 \mathrm{~cm}$ depth) is close to the annual accumulation at NorthGRIP (19.5 cm ice equivalent), the seasonal variability will, to first order, be averaged out in the experimental data. Still, some interannual variations in the impurity content of the ice are observed (Svensson and others, 2003b). These fluctuations may explain the small discrepancies between model and observations in Figures 2 and 3 , since the values of $D$ and $f$ are determined by the average concentration. We can take these fluctuations into account for the older samples, which have almost reached a steady state, by rescaling the crystal-size distributions. In Figure 4 we demonstrate that this rescaling results in a universal curve for all the size distributions, described by the steady-state Bessel function solution, which is significantly different from the widely used log-normal distribution. The "data collapse" in the figure, i.e. the fact that all the rescaled distributions have the same shape, clearly supports the oneparameter nature of the steady-state solution Equation (7).

By formulating Equation (2) we thus provide a comprehensive description of the dynamical processes of ice crystals. Our model improves the normal grain-growth law based on diffusive growth alone and offers an explanation of the reach of a steady state for the mean crystal size by means of fundamental physical processes. The good agreement between data and model suggests that polygonization/fragmentation is the dominant process in the production of small crystals, rather than nucleation of new crystals, a process not included in the model. The suggested interplay between the diffusion and the fragmentation in the crystal grain growth is believed to be an essential ingredient of many other systems in nature (e.g. geological and perhaps biological processes; see Ferkinghoff-Borg and others, 2003) and could be a useful tool to predict the distribution curves of fragmented pieces.

\section{REFERENGES}

Alley, R. B. and G. A. Woods. 1996. Impurity influence on normal grain growth in the GISP2 ice core, Greenland. F. Glaciol., 42(141), 255-260.

Alley, R. B., J. H. Perepezko and C. R. Bentley. 1986. Grain growth in polar ice: II. Application. 7. Glaciol., 32(112), 425-433.

Alley, R. B., A.J. Gow and D. A. Meese. 1995. Mapping $c$-axis fabrics to study physical processes in ice. F. Glaciol., 41(137), 197-203.

Dahl-Jensen, D. and 6 others. 1998. Past temperatures directly from the Greenland ice sheet. Science, 282(5387), 268-271.

Dahl-Jensen, D. and 8 others. 2002. The NorthGRIP deep drilling programme. Ann. Glaciol., 35, 1-4.

Ferkinghoff-Borg, J., M. H. Jensen, J. Mathiesen, P. Olesen and K. Sneppen. 2003. Competition between diffusion and fragmentation: an important evolutionary process of nature. Phys. Rev. Lett. 26. (10.1103/ PhysRevLett.91.266103.

Gow, A. J. and 6 others. 1997. Physical and structural properties of the Greenland Ice Sheet Project 2 ice cores: a review. F. Geophys. Res., 102(C12), 26,559-26,575.

Johnsen, S. J. and 8 others. 2001. Oxygen isotope and palaeo-temperature records from six Greenland ice-core stations: Camp Century, Dye-3, GRIP, GISP2, Renland and NorthGRIP. f. Quat. Sci., 16(4), 299-307.

Li Jun, T. H. Jacka and V. Morgan. 1998. Crystal-size and microparticle record in the ice core from Dome Summit South, Law Dome, East Antarctica. Ann. Glaciol., 27, 343-348.

Louat, N. P. 1974. On the theory of normal grain growth. Acta Metall., 22, 721-724.

Paterson, W. S. B. 1994. The physics of glaciers. Third edition. Oxford, etc., Elsevier.

Svensson, A. and 6 others. 2003a. Properties of ice crystals in NorthGRIP late- to middle-Holocene ice. Ann. Glaciol., 37, 113-118.

Svensson, A., P. Baadsager, A. Persson, C. S. Hvidberg and M.-L. SiggaardAndersen. 2003b. Seasonal variability in ice crystal properties at NorthGRIP: a case study around $301 \mathrm{~m}$ depth. Ann. Glaciol., 37, 119-122.

Thorsteinsson, T. 2002. Fabric development with nearest-neighbour interaction and dynamic recrystallization. 7. Geophys. Res., 107(B1), ECV3-1 to ECV3-13. (10.1019/2001JB000244.)

Thorsteinsson, Th., J. Kipfstuhl and H. Miller. 1997. Textures and fabrics in the GRIP ice core. f. Geophys. Res., 102(C12), 26,583-26,599.

Wang, Y., T. Thorsteinsson, J. Kipfstuhl, H. Miller, D. Dahl-Jensen and H. Shoji. 2002. A vertical girdle fabric in the NorthGRIP deep ice core, North Greenland. Ann. Glaciol., 35, 515-520. 